\title{
Effect of part sweep on axial flow compressor performance in the presence of circumferential casing grooves
}

\author{
SHRAMAN NARAYAN GOSWAMI ${ }^{1, *}$ and M GOVARDHAN ${ }^{2}$ \\ ${ }^{1}$ Honeywell Technology Solutions, Devarabisanahalli, Bangalore 560103, India \\ ${ }^{2}$ Department of Mechanical Engineering, Indian Institute of Technology Madras, Chennai 600036, India \\ e-mail: shraman.goswami@honeywell.com; gova@iitm.ac.in
}

MS received 4 December 2018; revised 17 April 2019; accepted 21 June 2019

\begin{abstract}
Modern axial compressors demand high performance and increased operating range. High performance is generally obtained by employing 3D design features, such as sweep and lean. To improve operating range, use of circumferential casing grooves is quite common. An extensive numerical study is carried out to understand performance change due to swept rotor blade on axial compressor performance and stall margin, in the presence of circumferential casing grooves. Numerical methodology used in the current work is validated with experimental data of NASA Rotor37. Grid sensitivity as well as turbulence model validation is carried out to validate numerical methodology used in this work. A baseline rotor is created without any sweep. Sweep considered in this study is employed only at part span of the blade. Impact of part sweep with circumferential casing grooves is not reported by many in open literature, which is the focus of this work. Different magnitudes of sweep are considered in this study. The current study indicates existence of an optimum combination of magnitude of sweep and span location at which sweep starts from. Sweep in the presence of circumferential grooves results in considerable increase of operating range with nominal decrease in efficiency. A detailed flow field investigation is presented to understand the underlying flow physics.
\end{abstract}

Keywords. Axial compressor; stall; part span sweep; casing treatment.

\section{Introduction}

Sweep and lean are the most common 3D blade design features used, to improve axial compressor performance. These design features help redistribute or increase loading as well as decrease secondary flow impact on main flow. Breugalmans [1] has carried out experimental study on a compressor cascade with NACA-65 series aerofoils. Different inlet boundary layer thicknesses are used on the endwalls. A number of different blade geometries are designed using three different stacking lines, namely straight line, circular and elliptical. He has reported that for a cascade, moderate magnitude of lean helps decrease secondary flow by reducing loading near endwalls. Sasaki and Breugalmans [2] in their study have noticed that near the forward part of the blade, forward sweep moves the flow towards endwall in the suction side and away near the pressure side. This is in the opposite direction of passage secondary flow and hence reduces cross-passage flow of endwall towards the suction side. This helps in reducing corner stall. Inoue et al [3] have observed performance improvement due to endwall controlling and its impact on secondary flow. They have designed

*For correspondence

Published online: 12 August 2019
Controlled-Endwall-Flow rotor blades and tested in a low-speed cascade. The rotor blades are designed with leading edge sweep. They have reported a marginal increase in efficiency but a large improvement in stall margin. Introducing sweep to an axial compressor blade changes flow incidence angle. This was reported by Godwin [4], for a compressor, designed to operate at low Mach number condition. It is also reported by Wadia et al [5] that sweep decreases axial flow diffusion as well as accumulation of the shroud boundary layer, which results in the reduction of interaction of shock and boundary layer. Performance gain achieved by swept blade is said to be due to these factors. In a numerical investigation by Govardhan and Ramakrishna [7], they have observed that lean, introduced by axial sweep, results in deflection of streamlines towards the endwalls and helps in energizing the endwall boundary layer. In a recent study, Biollo and Benini et al [8] have studied combined effect of lean and sweep on a geometry derived from Rotor37. Lean towards direction of rotation and sweep towards downstream rotor are reported to result in increased efficiency and pressure ratio, along with operating range. It is to be noted that the sweep applied is in the axial direction and hence also results in some lean. They have reported that shock structure in 
meridional plane is impacted by sweep whereas lean plays a major role in blade to blade plane.

Many types of casing treatments are used in an axial compressor for stall margin improvement. Among the most commonly used casing treatments are circumferential grooves. Bailey [9] did a detailed experimental study of casing grooves with different numbers of grooves along with different widths, depths and locations. It is reported that grooves near mid chord of an axial compressor blade has maximum impact on stall margin improvement. They have noted that there was no loss of performance with circumferential casing grooves. An experimental work reported by Donald et al [10] includes grooves on the shroud of inlet stage of a compressor. Axially skewed slot and blade-angled slot as well as circumferential grooves were studied by them. They have observed improved performance of rotor as well as stage and also significant improvement in stall margin with axially skewed grooves. They have also noted that the improvement in performance is due to the fact that axial grooves are not extended beyond the blade edges. Tip recess with casing treatment is studied by Khan et al [12]. They have reported that impact of tip recess on stall margin improvement is much smaller than that of casing grooves. They have also observed that impacts of first two grooves are much higher than those of downstream grooves on improving stall margin. Improvement in stall margin is reported to be due to breakdown of tip vortex by casing grooves. In a study by Lin et al [11], they have used slot type of grooves. Radially inclined axial slots are studied in this study. They have positioned the slots in such a way that the middle of the slots is aligned with leading edge of the rotor blades. They have observed that at low speed there is an exchange of momentum between regions of high and low momentum flow, augmented by the presence of slots. Close to design speed, reservoir-like behaviour is observed in the slot. This flow phenomenon results in reduction of tip vortex and improved stall margin.

To understand change in flow field due to sweep in the presence of circumferential grooves, a numerical study is carried out. Effect of this design features on performance and stall margin is assessed. The numerical process used in this work is validated using NASA Rotor37 experimental data. For the current study, the baseline rotor is derived from NASA Rotor37. The baseline rotor is designed to have no sweep or lean, but choke flow and pressure ratio are maintained at the same level as those of NASA Rotor37. Numerical investigations are carried out for different swept rotors. These simulations are carried out with five circumferential grooves. The baseline rotor is analysed with smooth shroud as well as five grooved shrouds. Unlike most of the previous studies, sweep does not extend from hub to tip, but starts at different spans of the blade.

\section{Numerical methodology validation}

Rotor37 experimental data $[13,14]$ are used for validation of numerical methodology used in this study. Extensive investigation of this rotor is carried out by many researchers [15-20]. The operating condition, for which this rotor is designed, makes it suitable to be used for validation. The characteristic geometrical values as well as design point performance values of this rotor are given in table 1. Numeca AutoGrid5®version 9.0-2 is used to generate structured mesh along with matching groove mesh. Details about numerical methodology are presented in already published work by current authors [21]. To obtain meshindependent results from the numerical analyses, mesh sensitivity study is carried out with three different mesh sizes (1.5 million, 2.5 million and 3.0 million). All the results reported here are with 2.5 million mesh count, as 3.0 million mesh count did not show any improvement over 2.5 million case. Minimum skewness obtained for the final mesh is approximately equal to 20 degrees. The final mesh used for comparison of numerical results to experimental data is shown in figure 1 [21]. Ansys CFX®version 15.0 is used to carry out CFD analysis (single passage, steady, RANS). Figure 1 [21] presents a qualitative description of applied boundary conditions.

The numerical stall point is considered as the maximum back pressure for which numerically stable result can be obtained. Beyond this maximum back pressure, an increase of back pressure by $0.01 \mathrm{psi}(69 \mathrm{~Pa})$ results in numerically unstable solution. It is understood that the steady numerical model applied in this current study does not have the fidelity to predict exact real stall point. However the last numerically stable point can be considered as an indicative of approaching flow break down. Hence, this point can be considered as close to aerodynamic instability of the compressor in reality [8].

In numerical analyses, turbulence models play a significant role. In order to find out the most suitable turbulence models for the current study, turbulence model validation is

Table 1. Performance parameter of NASA Rotor37.

\begin{tabular}{lc}
\hline Hub-to-tip diameter ratio at inlet & 0.7 \\
Blade aspect ratio & 1.19 \\
Inlet Mach number (relative) at tip & 1.48 \\
Inlet Mach number (relative) at hub & 1.13 \\
Tip solidity & 1.29 \\
Blade aerofoil sections & $\mathrm{MCA}$ \\
Fillet radius & $2.5 \mathrm{~mm}$ \\
Corrected speed & $1800 \mathrm{rad} / \mathrm{s}$ \\
Tip speed & $454.1 \mathrm{~m} / \mathrm{s}$ \\
Corrected mass flow & $20.19 \mathrm{~kg} / \mathrm{s}$ \\
Total pressure ratio & 2.106 \\
Polytropic efficiency & $88.9 \%$ \\
Number of blades & 36 \\
\hline
\end{tabular}




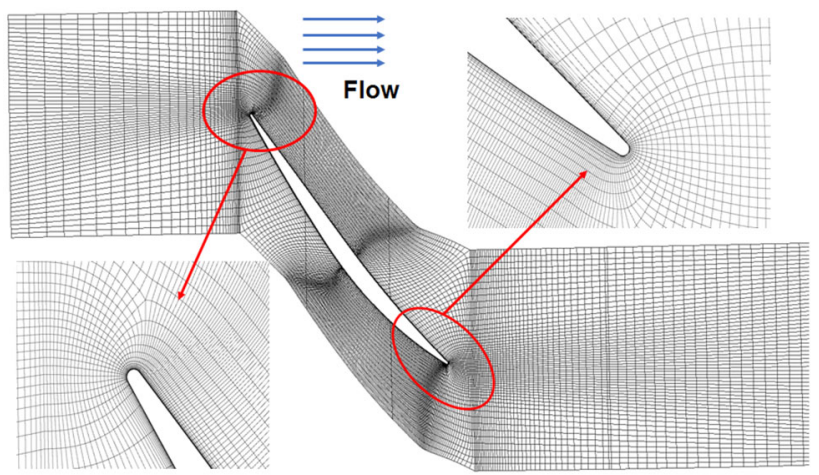

Mesh at $\mathbf{5 0 \%}$ Span

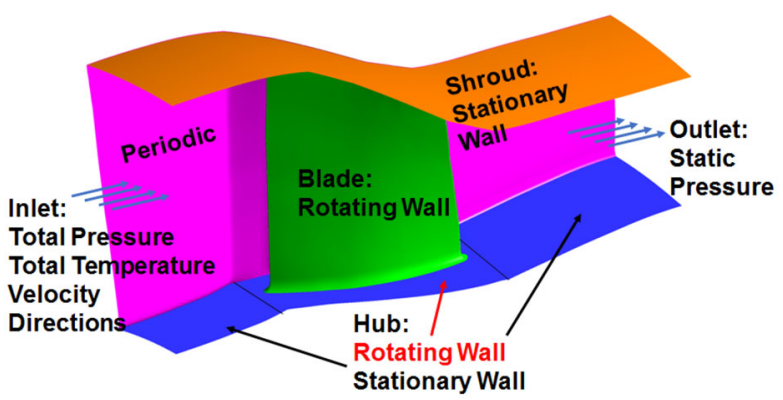

Boundary Conditions

Figure 1. Mesh and boundary conditions used for validation.

carried out. Turbulence model validation is carried out using three turbulence models [22], namely, Shear Stress Transport (SST), SST-Reattachment and Baseline k-Omega (BSL). Total pressure ratio vs corrected mass flow, $M c$, is compared and presented in figure 2. Out of the three turbulence models, numerical results obtained from BSL turbulence model is found to have the closest match with

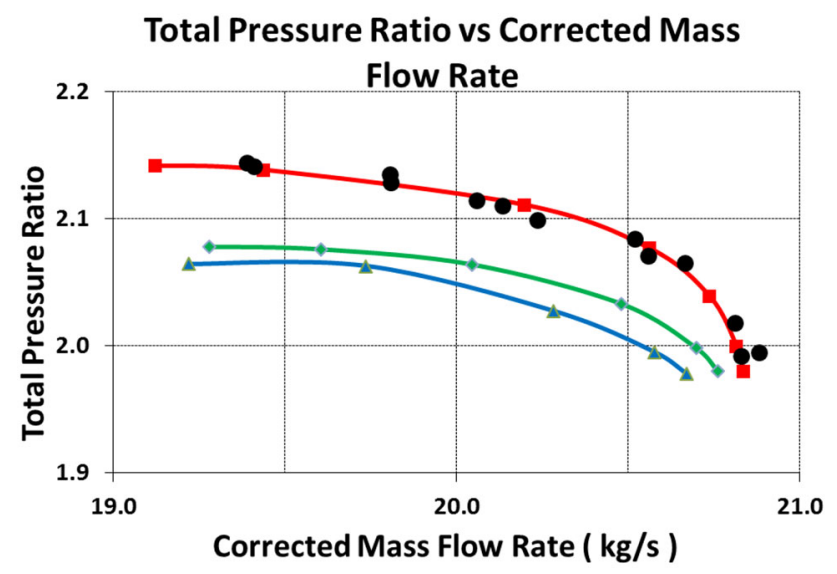

- Experimental Data $\rightarrow$-BSL $\rightarrow-S S T \rightarrow-S S T R M$

Figure 2. Comparison of turbulence models [8].

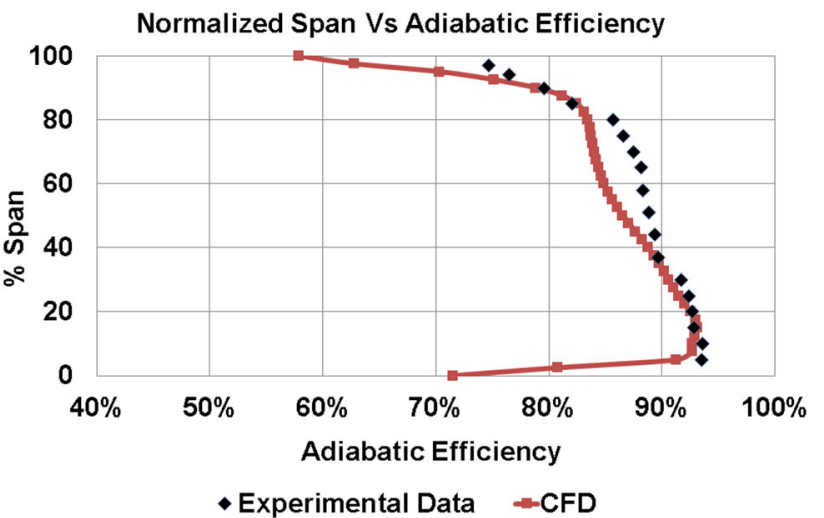

Figure 3. Span-wise variation of adiabatic efficiency [8].

experimental data. Figure 3 compares hub to shroud variation of efficiency. A good agreement between numerical and experimental data is obtained and hence a similar numerical process is followed in the rest of the study.

Corrected mass flow is defined as

$$
M c=M \sqrt{\frac{\left(\frac{T T_{\text {in }}}{288.17}\right)}{\left(\frac{P T_{\text {in }}}{101324.6}\right)} .}
$$

Efficiency is defined as

$$
\text { efficiency }=\frac{P R^{\left(\frac{\gamma-1}{\gamma}\right)}-1}{T R-1} .
$$

Averaged quantities are computed as mass flow averaged. Mass flow averaging of any variable, $\phi$, is defined as

$$
\bar{\phi}=\frac{\Sigma \phi_{\mathrm{i}} m_{\mathrm{i}}}{\Sigma m_{\mathrm{i}}} .
$$

\section{Baseline rotor design}

One of the most representative rotors of modern axial compressor is NASA Rotor37. Hence, baseline rotor geometry is derived from NASA Rotor37 geometry, by removing lean and sweep from it. It is intended that the baseline rotor should have a similar choke flow as that of NASA Rotor37 as well, as it should be able to develop the same or higher pressure ratio than that of NASA Rotor37, at design speed. Design process started by taking only the hub and shroud profiles from NASA Rotor37. These two profiles are then radially stacked through the centre of gravities of these profiles to remove lean and sweep from the rotor. Flow path is retained to be the same as NASA Rotor37 flow path. Numerical analyses of this rotor have predicted a lower mass flow than that of NASA Rotor37 choke flow as the throat gets reduced when profiles are radially stacked through centre of gravity. In order to increase flow rate, a number of design changes are made. 


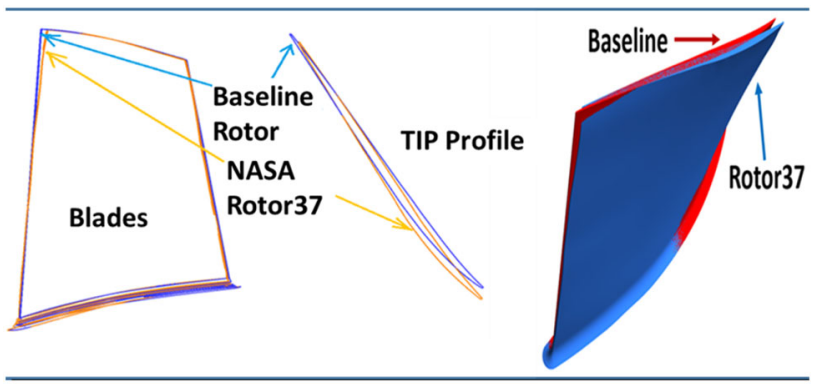

Total Pressure Ratio vs Corrected Mass Flow Rate

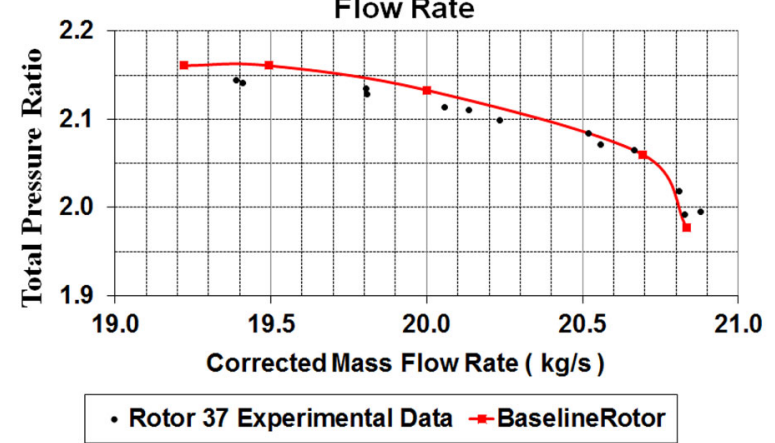

Figure 4. Geometry and performance comparison of Rotor37 and baseline rotor [8].

The design that results in similar mass flow as that of NASA Rotor37 is obtained by increasing the throat area. The tip profile of the new blade is rotated by 1.73 degrees about stacking axis to gain extra mass flow in choke. Qualitative comparison of geometry of baseline rotor and NASA Rotor37 and comparison of total pressure ratio of these rotors are presented in figure 4 .

\subsection{Baseline rotor with casing grooves}

It is already understood that sweep influences flow near endwalls. In such a scenario, the presence of casing grooves on the shroud can impact the endwall flow. To understand this interaction of sweep and circumferential groove, the rotor shroud is modelled with five numbers of circumferential grooves. A number of researchers have worked on finding optimum number of grooves. It is reported [6] that five grooves are found to be optimum for Rotor37 and hence five grooves are chosen for this study. The groove mesh is made to have one to one match with main flow passage. Details of mesh and geometry of the grooves are shown in figure 5. To find the impact of casing grooves on the baseline rotor performance, numerical analyses of this rotor are carried out with and without casing grooves. Average $Y$-plus values obtained on blade, hub and shroud surfaces are 2.7, 2.1 and 1.8, respectively. Average $Y$-plus on the groove surfaces is close to 8 . Convergence criteria used are that all imbalances (mass, momentum and energy) are lower than $\pm 0.01 \%$. Figure 6 shows the efficiency comparison with and without casing grooves on baseline
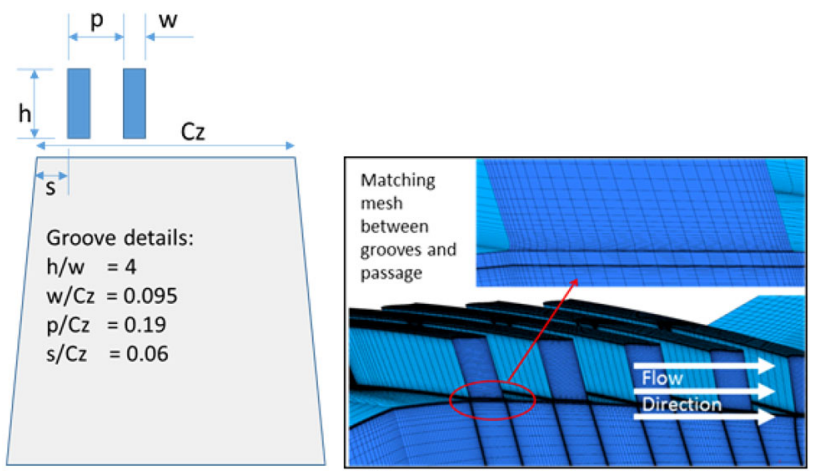

Figure 5. Groove geometry and 1:1 matching mesh between main passage and grooves.

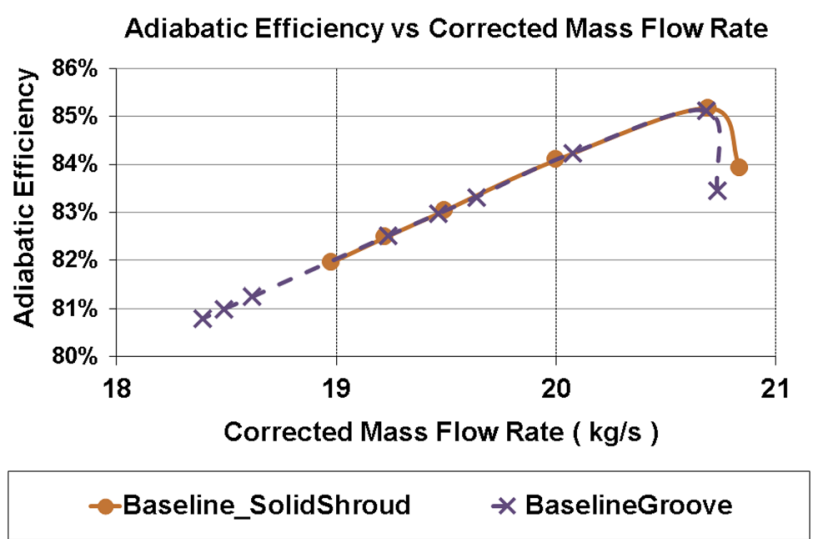

Figure 6. Variation of adiabatic efficiency for baseline rotor with and without groove.

rotor. Although efficiency and pressure ratio are close for the two cases, the grooves have resulted in stall margin increase by $3.72 \%$. Definition used for stall margin in the current study is as follows:

$$
\text { stallmargin }=\frac{\left(\frac{P R}{M}\right)_{\mathrm{stall}}}{\left(\frac{P R}{M}\right)_{\mathrm{OP}}}-1 .
$$

\section{Rotor blade design with sweep}

Rotors with different sweeps are generated to study impact of sweep on different performance parameters. There are different definitions of sweep used in literature. Some of the researchers have used sweep in the chordwise direction, whereas a number of other researchers have used axial sweep to study the effect on performance. It is reported that axial sweep results in induced lean [7]. To segregate the effect of lean and sweep, sweep is applied in the direction of chord. Eighteen swept geometries are created using $\pm 5 \%, \pm 10 \%$ and $\pm 15 \%$ of tip chord of the rotor. In order to study impact of sweep start location, sweeping is done from various span-wise locations such as, 50\%, 75\%, 85\% 
Table 2. Magnitude and starting span of swept blades.

\begin{tabular}{lc}
\hline Sweep start span $(\%)$ & Sweep magnitude $(\%)$ \\
\hline 50 & $\pm 5, \pm 10$ and \pm 15 \\
75 & $\pm 5, \pm 10$ and \pm 15 \\
85 & $\pm 5, \pm 10$ and +15 \\
90 & \pm 5 \\
\hline
\end{tabular}
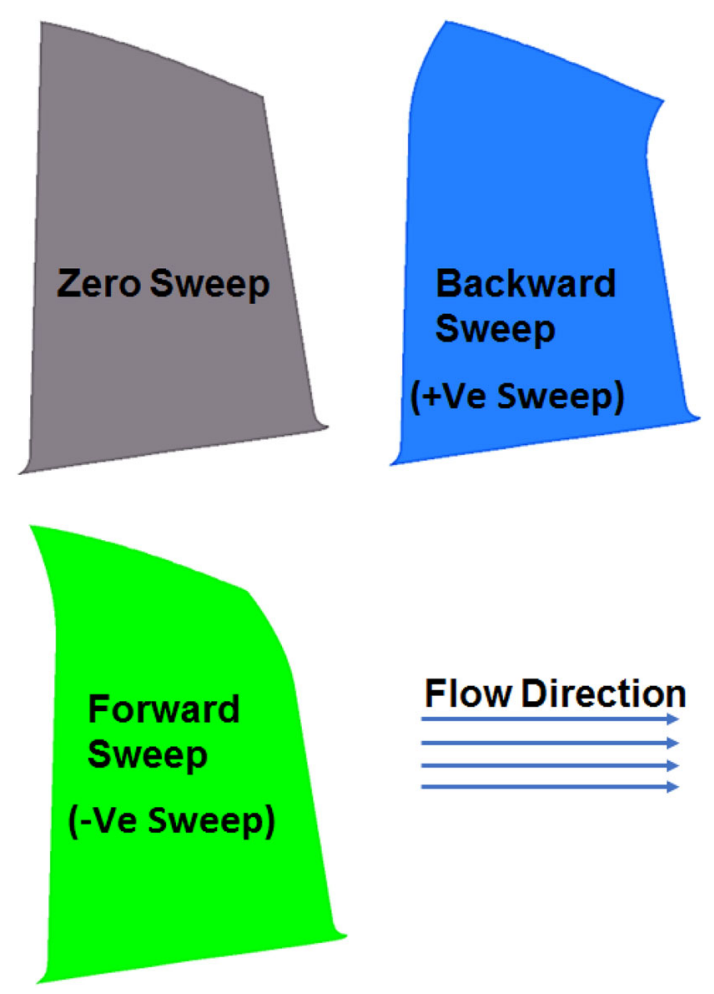

Figure 7. Representative rotor geometries with sweep.

and $90 \%$ span (table 2). Figure 7 shows representative rotor geometries with sweep. Magnitude of sweep, $\lambda$, is presented as \%chord such that

$$
\% \text { sweep }=\frac{\lambda}{C h} \times 100 .
$$

Positive value of sweep indicates backward sweep and negative value of sweep indicates forward sweep. The relative position of grooves with respect to blade leading edge is maintained to be the same, by moving the grooves in axial direction.

Table 3 summarizes performance parameters of all the rotors. From the data it is observed that rotor geometry with "+5\% sweep, 75\%span" has the lowest numerically stable mass flow. The following section discusses the results obtained in this study.

\section{Results and discussion}

It is observed from table 3 that most of the forward swept blades have resulted in higher pressure ratio than that of the baseline rotor. For forward swept rotors, as sweep starting span is increased, there is a drop in pressure ratio. For example, for $10 \%$ forward swept blades, pressure ratio is higher for the case where sweep starts at $50 \%$ span, as compared with $75 \%$ and $85 \%$ sweep starting span. For sweep starting span at 50\%, all the three forward swept cases $(-5 \%$, $-10 \%$ and $-15 \%$ ), pressure ratio is higher than that of the baseline rotor. It can also be observed that for a particular sweep start span location, as magnitude of forward sweep increases, pressure ratio also increases. Combining these two observations, it can be inferred that, to get a higher pressure ratio, sweep start span needs to be decreased as well as magnitude of forward sweep needs to be increased. On the other hand, for backward swept blades, higher sweep starting span has resulted in better pressure ratio. Data also reveal that impact of sweep on stall margin improvement is higher for all backward swept blades. Only for sweep start span $50 \%$ with $5 \%$ forward sweep, a slightly higher stall margin is observed than the baseline case; 5\% backward sweep has resulted in better stall margin than $10 \%$ backward sweep for sweep starting span 50\% and 75\%. For 85\% sweep start span case, $10 \%$ backward sweep has resulted in higher stall margin. It is also observed that with increase in sweep start span, for better stall margin, magnitude of backward sweep needs to be increased. Increase or drop in efficiency is found to be marginal for the design changes used in this work. Difference between maximum and minimum values of maximum efficiency is found to be $0.9 \%$, although change in stall margin is from $+8.9 \%$ to $-4 \%$. The maximum and minimum efficiencies are obtained, respectively, for the 5\% forward and 5\% backward swept blades with sweep start span 50\%. Sweep start span 50\% results in the top three highest efficiencies, although stall margin is decreased for $10 \%$ and $-15 \%$ swept blades.

In a similar line of findings by a few other researchers $[23,24]$, for grooved cases, reduction in choke flow is observed in the current study too. In order to understand the reason for the decrease of choke flow capacity, mass flux at rotor trailing edge is compared for baseline rotor with solid shroud and grooved shroud.

Mass flux can be expressed in terms of density and meridional velocity as follows:

$$
\begin{gathered}
\text { massflow }=\rho V_{\mathrm{m}} A, \\
\text { massflux }=\frac{\text { massflow }}{A}=\frac{\rho V_{\mathrm{m}} A}{A}=\rho V_{\mathrm{m}} .
\end{gathered}
$$

Mass flux variation from hub to shroud at near-choke condition for baseline rotor, with and without casing grooves, is shown in figure 8 . Near the tip region, lower mass flux is observed for grooved rotor, which results in choke flow reduction. The lower mass flux near the blade 
Table 3. Summary of performance parameters.

\begin{tabular}{lccccccc}
\hline $\begin{array}{l}\text { Sr. } \\
\text { no. }\end{array}$ & $\begin{array}{c}\text { Sweep } \\
\text { magnitude }(\%)\end{array}$ & $\begin{array}{c}\text { Sweep } \\
\text { start span }(\%)\end{array}$ & $\begin{array}{c}\text { PR } \\
\text { @MaxEff }\end{array}$ & $\begin{array}{c}\text { Max. } \\
\text { efficiency }\end{array}$ & $\begin{array}{c}\text { Choke } \\
\text { flow (kg/s) }\end{array}$ & $\begin{array}{c}\text { Stall } \\
\text { flow }(\mathrm{kg} / \mathrm{s})\end{array}$ \\
\hline 1 & 0 & 0 & 2.056 & 85.17 & 20.83 & 18.98 & 13.26 \\
2 & +5 & 50 & 2.046 & 84.92 & 20.70 & 18.22 \\
margin $(\%)$
\end{tabular}

tip can be attributed to the interaction of tip vortex and casing grooves. It can also be noted that mass flow for swept and unswept blades are very similar when casing grooves are present.

\subsection{Impact of sweep on performance}

It is observed that blades with sweep starting span at $50 \%$ and having - ve sweep (forward sweep) result in higher efficiency near design point. Choke mass flow is found to be higher for these cases as compared with baseline rotor. Highest choke flow is found to be for " $-15 \%$ sweep, $50 \%$ span" case. Increase in efficiency is close to $1 \%$ for these cases as compared with baseline cases (with or without grooves). The increase in efficiency is associated with a loss in stall margin for this rotor. Stall margin is reduced by approximately $4 \%$ for this rotor as compared with baseline rotor without grooves. Lowest mass flow is observed for " $+5 \%$ sweep, $75 \%$ span" case (table 3). Stall margin for this case is found to be $22.1 \%$. As compared with baseline solid shroud and grooved shroud, this translates to an increase of stall margin by $8.9 \%$ and $4.6 \%$, respectively. This improvement is associated with a reduction in choke flow by $0.4 \%$ and efficiency by $0.2 \%$. Figure 9 shows the change in stall margin for different rotor geometries. Existence of an optimum value of sweep between $+5 \%$ to $+10 \%$ sweep can also be observed from this figure, which results in maximum stall margin improvement.

5.1a Flow field near tip region: In order to understand flow field near the tip region, flow structure at $98 \%$ span is compared and presented in figure 10, in terms of relative velocity and streamlines. The two cases compared here are baseline rotor with smooth shroud and swept rotor with smooth shroud, near lowest mass flow condition of baseline rotor $(19 \mathrm{~kg} / \mathrm{s})$. As marked in the figure, leading edge of swept rotor is at a distance ' $A$ ' downstream of unswept rotor leading edge. Similarly, distance marked ' $B$ ' is the distance by which separation bubble appears downstream in case of the swept rotor. It is noted that these two distances are very close to each other. Hence, although from flow path perspective, location of separation is downstream for swept rotor, in terms of blade relative stream location, there is no change in starting location of separation bubble. However, the size of separation bubble is larger for baseline rotor as compared with that of swept rotor. Also there is flow recirculation observed in baseline rotor at this location, which is not present in swept rotor flow field. This separation bubble and related recirculation zone is due to the shock on the suction side of the blade. As can be seen from figure 12, the gradient of pressure jump is more for the baseline rotor, which indicates a stronger shock. The separation bubble reduces in size at the lower span and increases at higher span, as shock strength decreases with decreasing radius.

Interaction of tip vortex with inter-blade passage flow is shown in figure 11. Six stream-wise planes are created from close to blade leading edge to blade trailing edge. These planes are created using normalized stream-wise locations $(28 \%, 35 \%, 45 \%, 55 \%, 65 \%$ and $73 \%)$ so that relative location of these planes with respect to blade leading edge remains the same. Contours of entropy are plotted on these planes. Also shown are streamlines from blade leading edge tip. The tip leakage vortex near blade leading edge mixes 


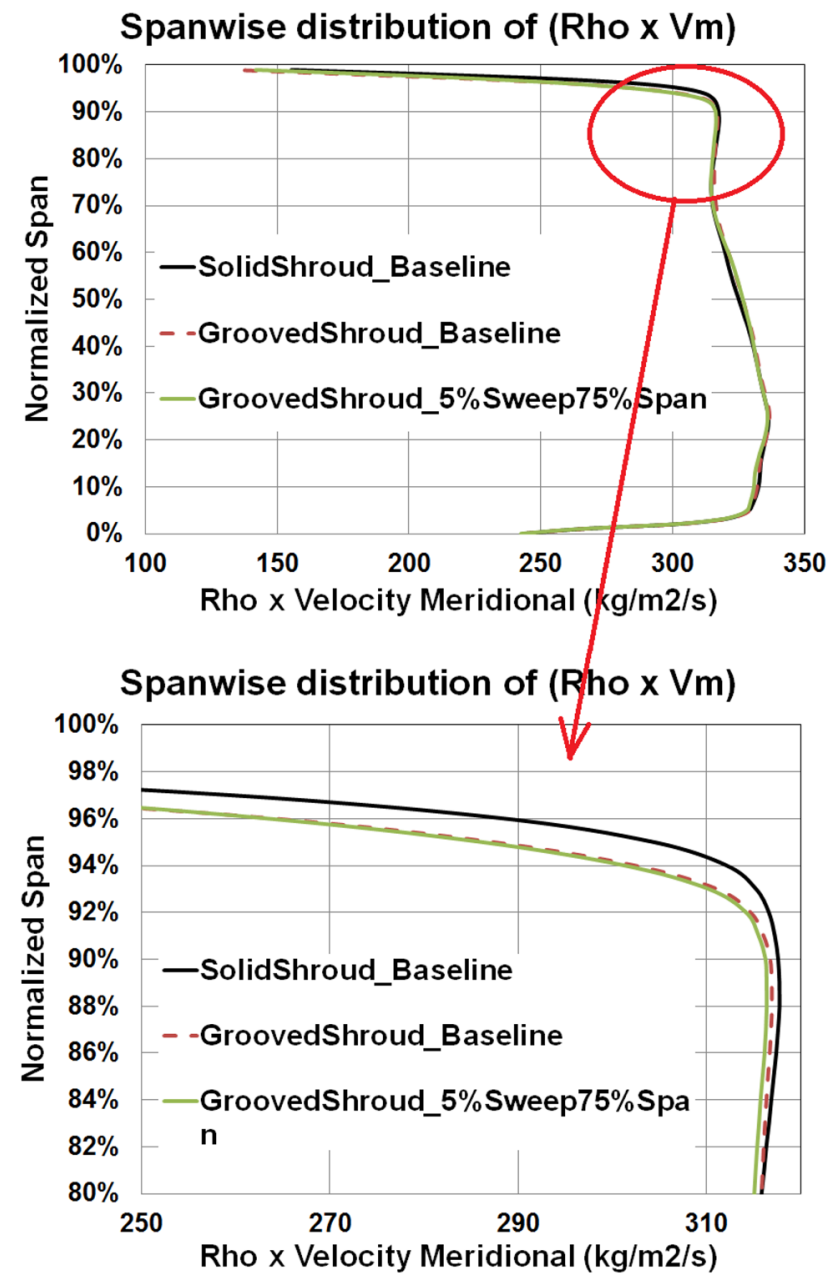

Figure 8. Mass flux comparison at rotor trailing edge.

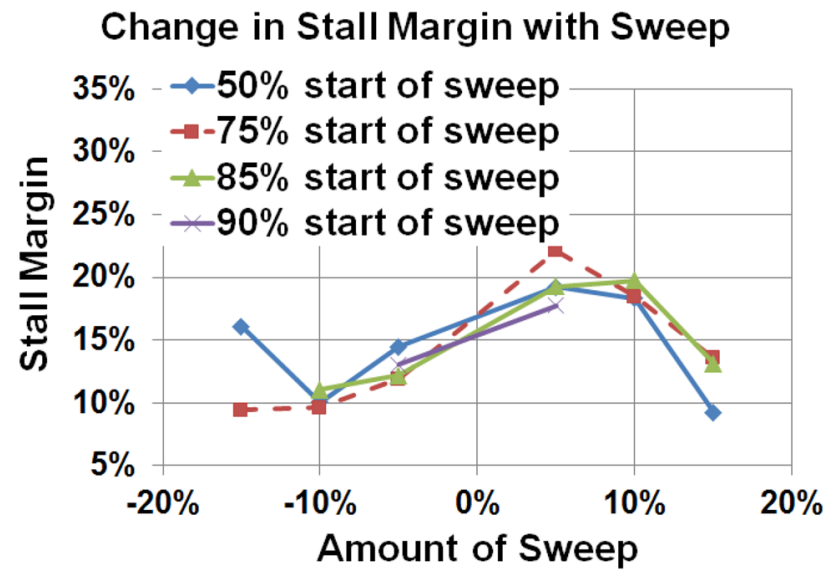

Figure 9. Stall margins of swept rotors.

with the passage flow (Plane P) and gradually gets convected towards the pressure side of the next blade (Plane $\mathrm{S}$ ). It can be observed that the interaction of tip vortex with

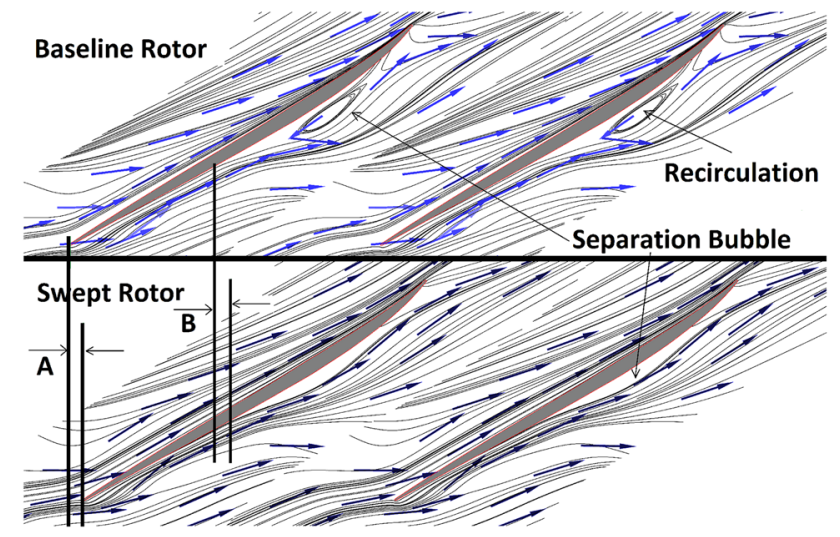

Figure 10. Velocity vector and streamline at $98 \%$ span.

passage flow results in the high entropy zone. The maximum entropy in case of baseline rotor is higher than in swept rotor, as can be seen in Plane R and Plane T. Also, comparing the size of vortex interaction region in these two planes, it can be inferred that, for the swept rotor the vortex interaction region with passage flow is larger than that of the baseline rotor, indicating a more diffused vortex in case of the swept rotor.

\subsection{Impact of sweep at constant sweep start span}

To understand impact of sweep magnitude, cases with sweep starting span at $75 \%$ are compared; $75 \%$ sweep start span cases are chosen for comparisons as we have observed highest stall margin improvements for swept blades for this sweep start span; $\pm 5 \%$ and $\pm 15 \%$ swept rotor results are compared for this study. For $-15 \%$ swept rotor, stall margin is the lowest but choke flow has increased (table 3). In comparison with baseline cases, $+5 \%$ swept rotor has resulted in highest increase in stall margin. It is observed that as sweep is changed from negative to zero, stall margin starts improving. A large increase in stall margin is observed as sweep is increased from $-5 \%$ to $+5 \%$. Stall margin starts to decrease as sweep is increased beyond $+5 \%$. Figure 12 shows zoomed in view of static pressure on blade surfaces at $75 \%$ and $99 \%$ span obtained for different rotors with sweep starting at $75 \%$ span. Stream-wise location $0 \%$ corresponds to blade leading edge and $100 \%$ corresponds to blade trailing edge in this figure. It can be observed that at $75 \%$ span, baseline rotor loadings are the same with smooth shroud and grooved shroud, whereas at $99.9 \%$ span, shock location on suction side is downstream for the grooved rotor. This is expected as impact of groove is not felt down to $75 \%$ span. This higher loading has helped in having higher stall margin for the grooved shrouded rotors. At $75 \%$ span, shock in case of backward swept rotors (+ve sweep) is found to be located downstream as compared with the forward swept rotors (-ve sweep). Shock locations move 


\section{Entropy Contour and Streamlines}
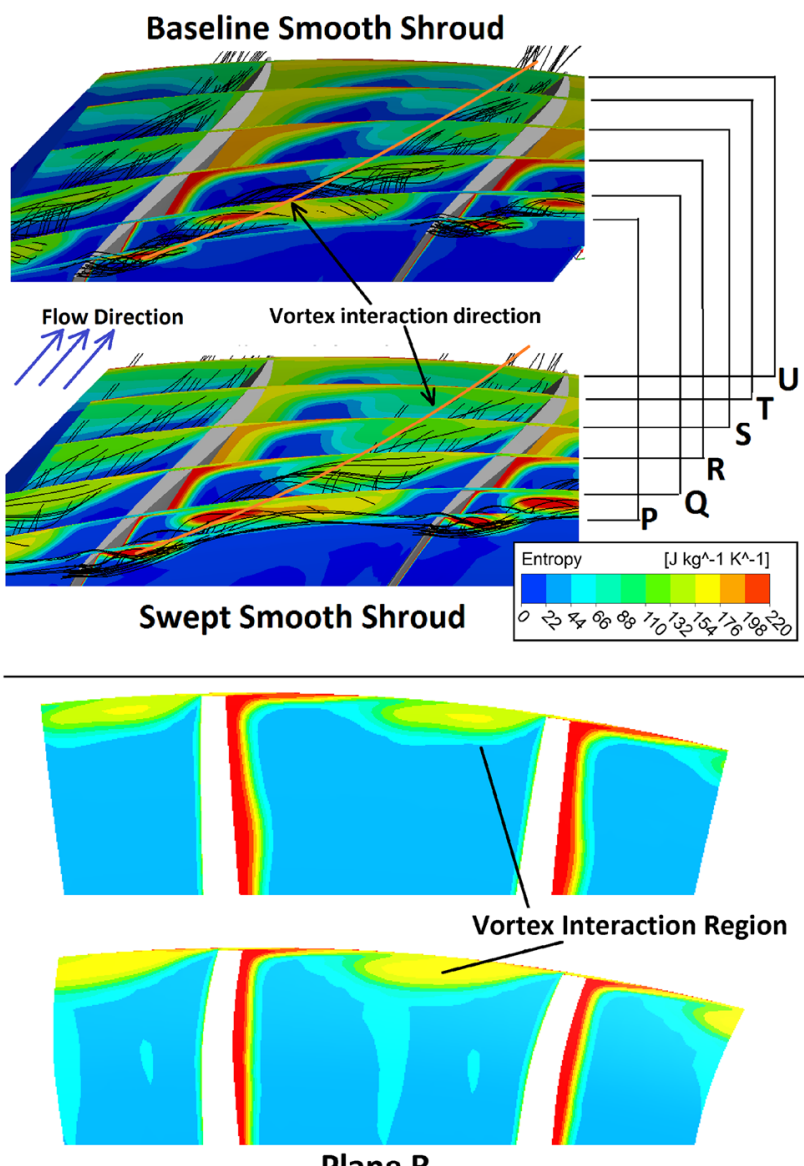

Plane R

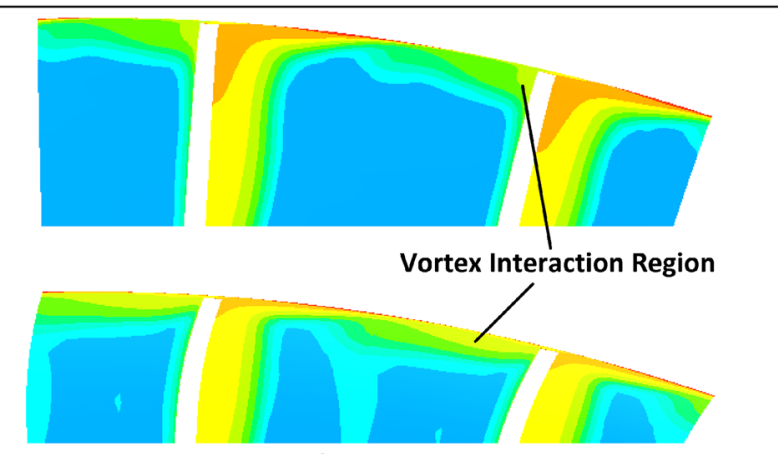

Plane T

Figure 11. Entropy contours and streamlines showing interaction of tip vortex and shock with main flow.

downstream as sweep is changed from $-15 \%$ to $+15 \%$. This results in increased loading as sweep is increased. Near the blade tip ( $99.9 \%$ span), this trend is reversed. This reversal in trend indicates existence of a span where difference in loading is similar between different swept rotors. It can also be observed that for backward swept rotors, pressure side pressure levels are higher. The zigzag pattern in loading chart near $99.9 \%$ span is due to the interaction of tip vortex and the grooves.
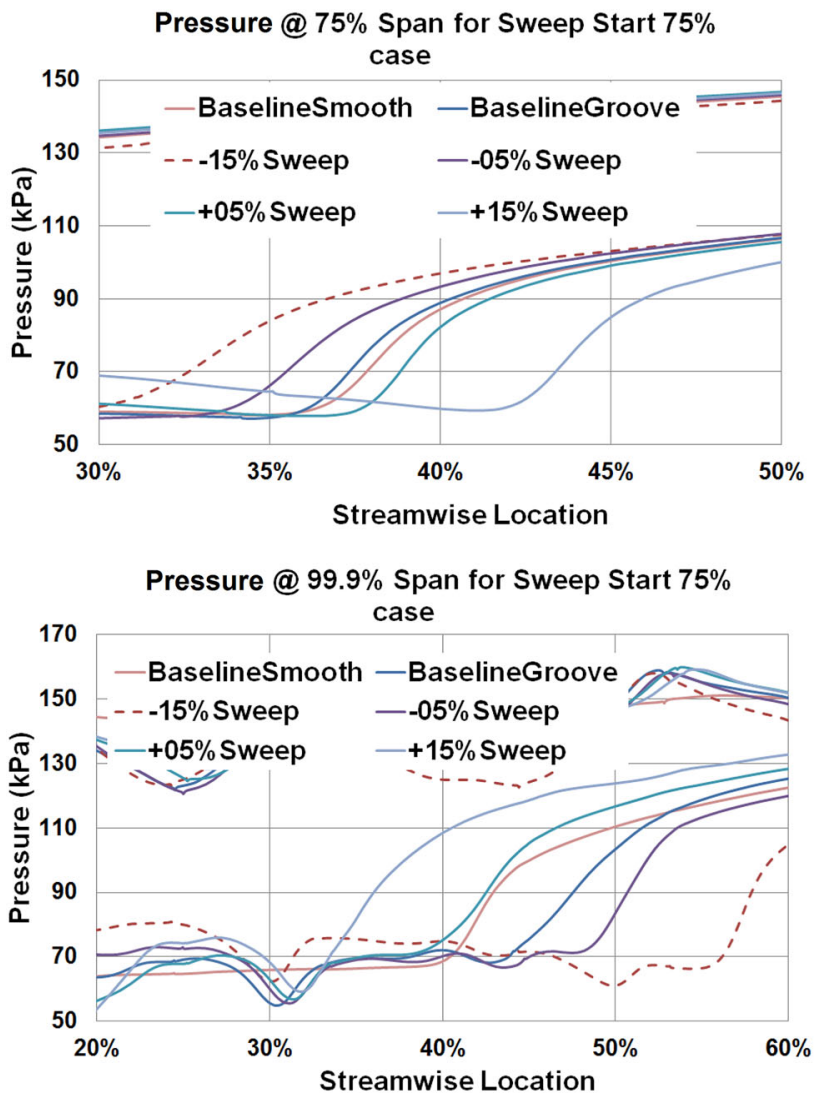

Figure 12. Pressure on blade surfaces at $75 \%$ and $99.9 \%$ span for $75 \%$ sweep start span.

\subsection{Effect of backward and forward sweep}

To understand the impact of backward and forward sweep, $+5 \%$ and $-5 \%$ swept rotors with grooves for sweep starting span $75 \%$ are compared near design flow $(20 \mathrm{~kg} / \mathrm{s})$. It is noticed that at $55 \%$ normalized stream-wise location, entropy for forward swept ( $-5 \%$ sweep) rotor is higher than that of backward swept ( $+5 \%$ sweep) rotor (figure 13$)$. To understand the reason for this increase in entropy, bladelimiting streamlines (blue coloured) and passage vortex (black colour) close to the blade suction surfaces are plotted in figure 13. The blade-limiting streamlines show different structures above $75 \%$ span, downstream of the shock location for the two cases. The trailing edge streamlines get split into three streams in case of positive swept blade and interact with the tip vortex. Also the passage vortex is larger for positive swept rotor than the negative swept rotor. This has resulted in a higher entropy at 55\% stream-wise location for forward swept rotor.

\subsection{Impact of sweep start location for constant magnitude of sweep on stall margin}

Four different sweep starting spans are considered for the same magnitude of rotor sweep $(+5 \%)$ to understand impact of sweep start location. Figure 14 shows the 


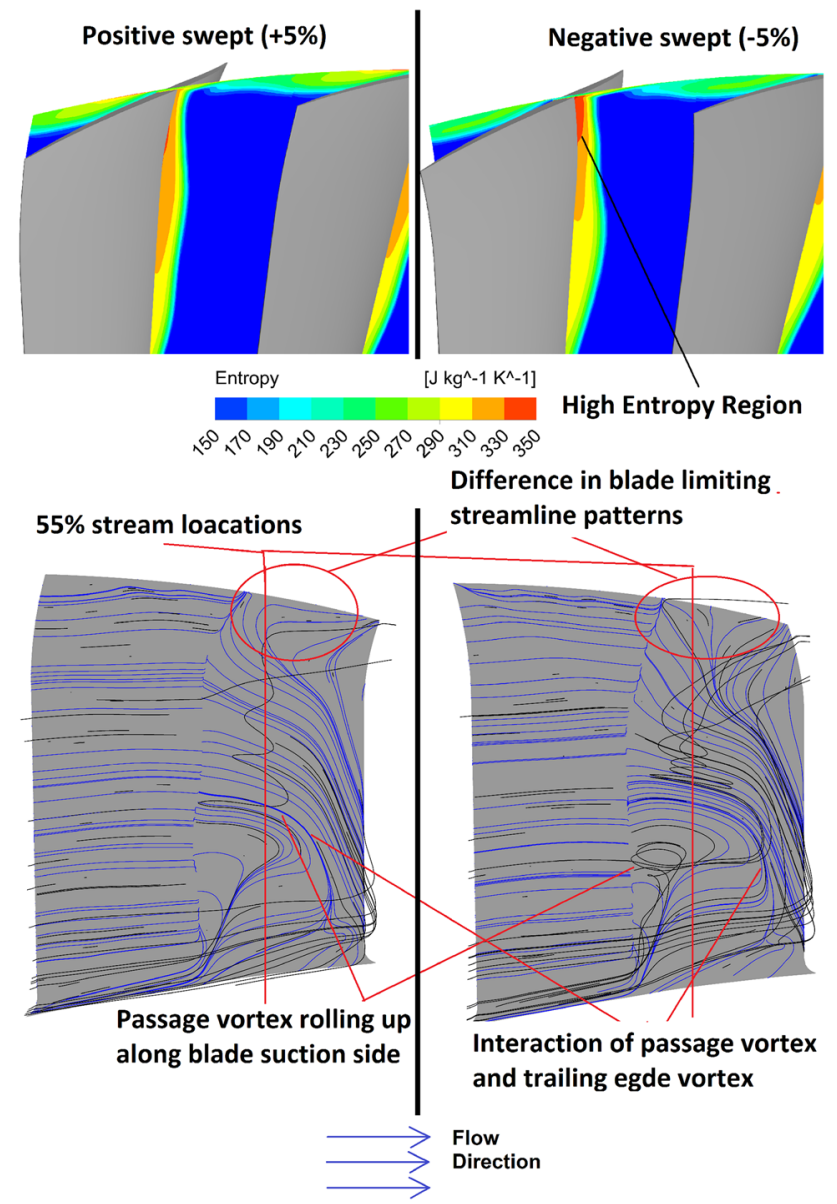

Figure 13. Entropy contours and streamlines in backward and forward swept rotors.

Total Pressure Ratio vs Corrected Mass Flow Rate

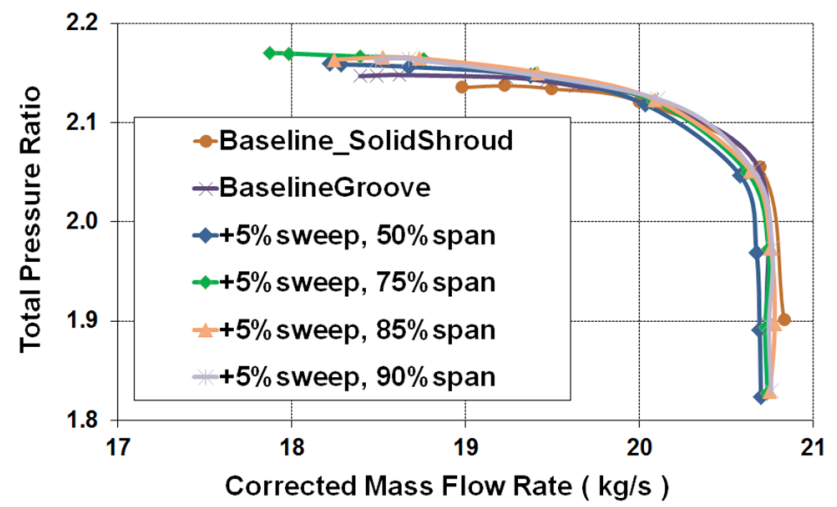

Figure 14. Effect of sweep start span for $+5 \%$ sweep on total pressure ratio.

comparison of total pressure ratio for these cases. Choke flow for all these cases are observed to be close to baseline grooved case but lesser than baseline solid shroud case. A significant stall margin improvement is observed as sweep starting span is changed from $50 \%$ to $75 \%$. As sweep start is moved to higher span, a decrease in stall margin is observed. The stall margin can become lower than that of baseline rotor if sweep start span is moved too high (in the current study, 90\% span case).

\subsection{Effect of groove on stall margin}

In order to compare effect of groove and effect of sweep on stall margin, baseline geometry with and without grooves is compared to " $+5 \%$ sweep, $75 \%$ span" geometry with and without grooves. Figure 15 shows comparative total pressure ratio plot of these cases; " $+5 \%$ sweep, $75 \%$ span" case with smooth shroud results in stall margin of $19.9 \%$, whereas for baseline rotor with smooth shroud, stall margin is $13.3 \%$. Hence, putting a swept blade has resulted in $6.6 \%$ increase in stall margin in a smooth shrouded compressor. Baseline rotor with groove has resulted in a stall margin of $17.5 \%$. By adding groove to the baseline rotor, an increase of $4.2 \%$ stall margin is realized. Stall margin for swept geometry with groove is $22.1 \%$. Increase in stall margin by sweeping the blade in the presence of groove is $4.6 \%$, whereas without groove the same swept blade results in an increase of stall margin by $6.6 \%$. Hence, it can be inferred that impact of sweep on stall margin improvement is more significant than that of grooves, for the current geometry.

5.5a Flow field near tip region Interaction of leading edge tip vortex with the flow through the first groove is shown in figure 16 . The cases compared here are baseline rotor with groove and $+5 \%$ sweep, $75 \%$ span rotor with and without grooves. The results correspond to the mass flow that is close to the lowest mass flow of baseline rotor with grooved shroud $(18.4 \mathrm{~kg} / \mathrm{s})$. The black coloured streamlines represent the leading edge tip vortex and red coloured streamlines represent flow through the first groove. For the smooth shroud case the streamlines are taken at the same location as in the grooved case. It is observed that for the grooved shroud cases (baseline as well as swept rotors) the leading edge tip vortex closely follows the blade suction

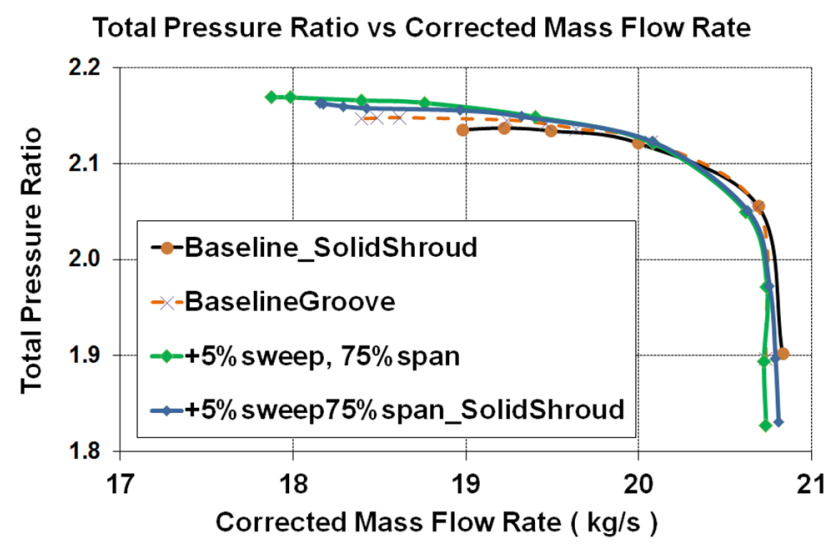

Figure 15. Effect of groove with and without sweep on total pressure ratio. 

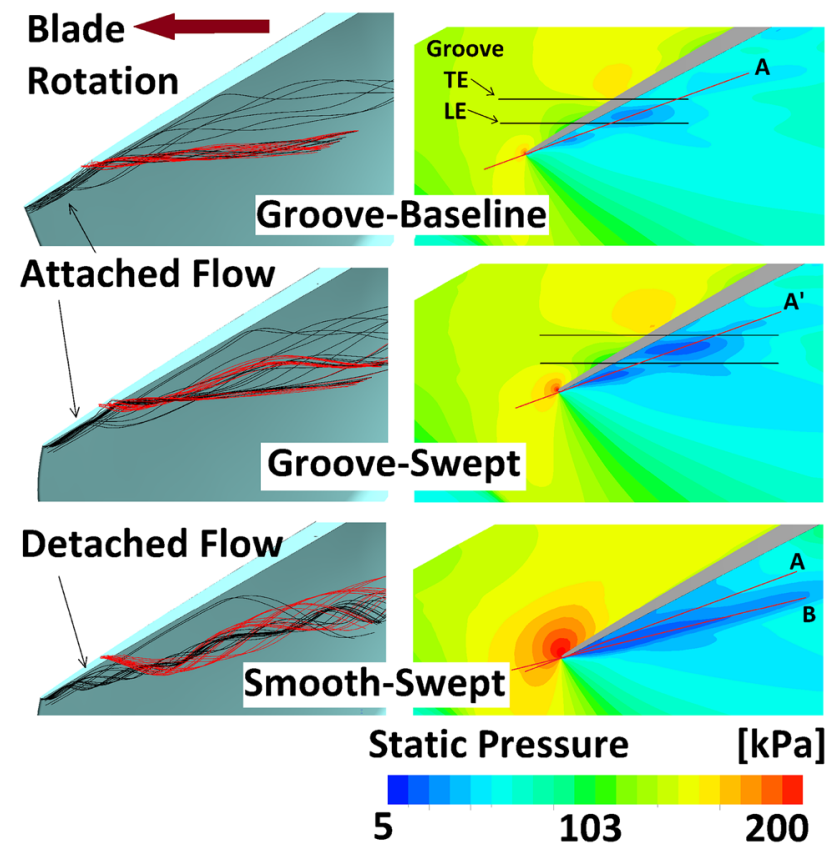

Figure 16. Leading edge tip vortex and first groove flow interaction: static pressure at tip for baseline rotor with groove and $+5 \%$ sweep, $75 \%$ span rotor with and without groove.

side up to the first groove, whereas for smooth shroud case it detaches from the suction side and merges with the flow over the tip coming through the groove. It is also noticed that direction of leading edge tip vortex for smooth shroud is more axial as compared with grooved shroud cases. Lines $\mathrm{A}, \mathrm{A}^{\prime}$ and $\mathrm{B}$ are drawn from the leading edge through the low static pressure zones (figure 16). The lines $\mathrm{A}$ and $\mathrm{A}^{\prime}$ are very close to each other but line $\mathrm{B}$ makes a larger angle with blade suction side as compared with that of A. The reason for these two observations can be attributed to the fact that the leading edge tip vortex gets sucked towards the low pressure zone created by the flow through the groove. This causes the leading edge tip vortex to flow close to the blade surface and hence an overall lesser turning away from the blade surface. This results in tip flow being more axial for grooved shroud case. Tip vortex being more axial indicates higher axial momentum, which helps in throttling the rotor to a lower mass flow rate (delayed stall). Flows through the five grooves are compared for baseline and swept shroud and presented in figure 17. The streamlines passing through the grooves are coloured by entropy. The overall structures of flow through the grooves for the two rotors are very similar. The flows through grooves three, four and five are almost identical. The flow inside first and second groove for baseline rotor has higher entropy than that of the swept rotor. This indicates a lower mixing loss near tip for swept rotor and hence results in a higher stall margin for swept rotor.

Magnitudes of flow, as percentage of inlet flow, through the tip gaps of baseline and swept rotors with and without grooved shroud are presented in figure 18. It can be noticed
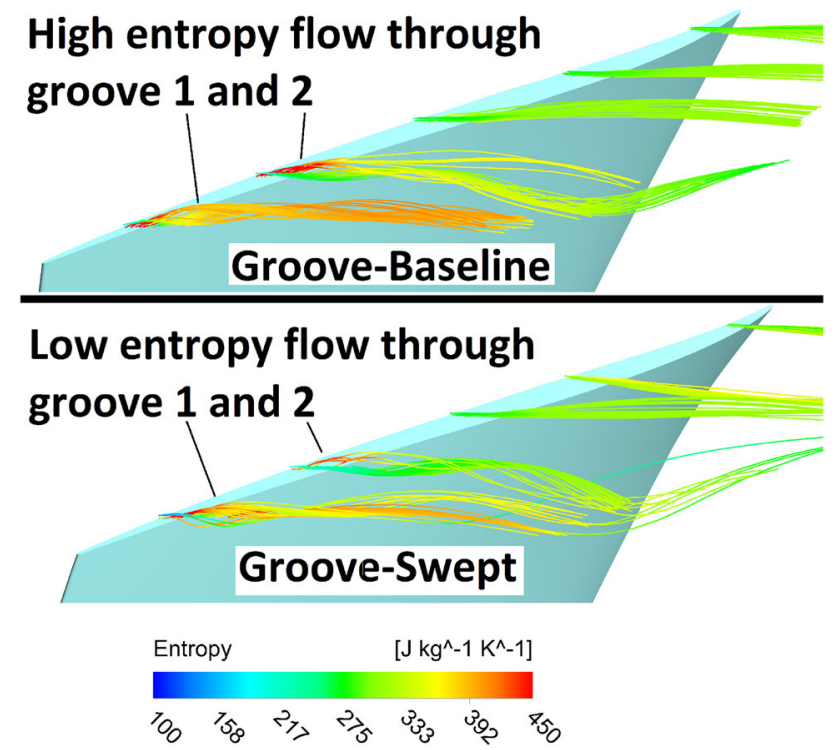

Figure 17. Flow through the grooves for baseline rotor and $+5 \%$ sweep, $75 \%$ span rotor.

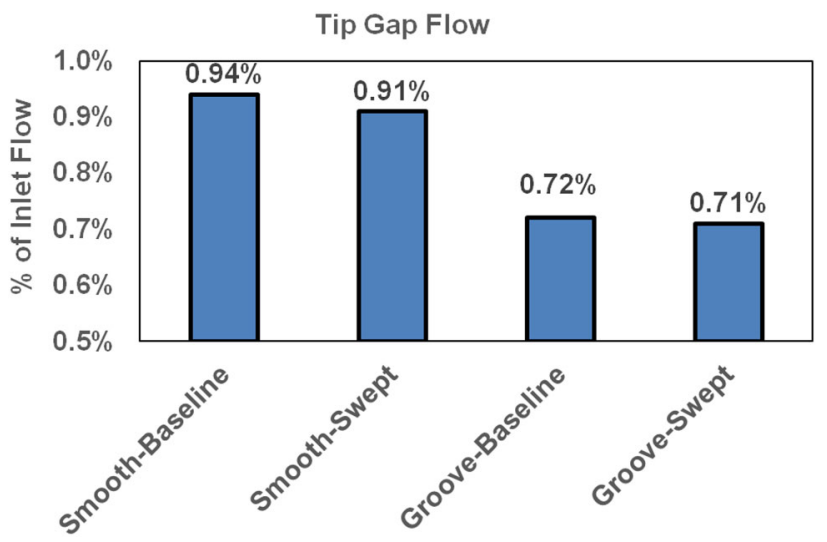

Figure 18. Variation of tip gap flow.

in case of solid shroud, as sweep is introduced to baseline rotor, that tip flow is reduced from $0.94 \%$ to $0.91 \%$ of the inlet flow. For the grooved case, as sweep is introduced, tip flow goes down from $0.72 \%$ to $0.71 \%$. However, as groove is introduced, baseline rotor tip flow reduces from $0.94 \%$ to $0.72 \%$ and swept rotor tip flow reduces from $0.91 \%$ to $0.71 \%$. Hence, it can be inferred that impact of groove on magnitude of tip leakage flow is more as compared with that of sweep. This is mainly due to the blockage created by the presence of grooves. Hence, it is understood that the performance of the swept rotor increases due to low loss tip leakage vortex as well as lower magnitude of tip leakage flow. Also it is noticed that impact of groove and sweep is more near the leading edge, which, for this case, extends till the second groove. 


\section{Conclusion}

A set of axial compressor rotor blades with sweep in the presence of smooth and grooved shroud are analysed and performance parameter as well as numerical stall is compared to those of radially stacked blades. The results from this study can be summarised as follows.

1. A decrease in choke mass flow is observed in rotors with casing grooves due to lower mass flux in the vicinity of blade tip. As a result of this mass flow shift, maximum efficiency point moves to that of a lower mass flow.

2. Pressure ratio drops with increase in sweep starting span and it increases with increase in magnitude of forward sweep. This trend is reversed for backward swept blades.

3. Flow migration as well as redistribution of loading is observed due to sweep. This has helped in stall margin improvement in backward swept rotor. On the other hand, for forward swept rotor, this has resulted in reduction in stall margin but increased choke flow.

4. There is a downstream migration of shock location on suction side of the rotor blade at 50\% span for forward swept rotors. Reverse trend is observed for these rotors at $99 \%$ span.

5. There exists an optimum sweep magnitude and sweep start location that result in maximum increase in stall margin. Stall margin decreases if sweep magnitude or sweep start span is increased or decreased.

6. Magnitude of stall margin improvement obtained using swept blades on a smooth shroud is more than using circumferential grooves.

7. The baseline rotor has low momentum zone near blade tip. Very small low momentum zone is observed for a swept rotor for the same mass flow condition as that of the baseline rotor.

8. Leading edge tip vortex gets pulled towards low pressure region created by the grooves. This results in a more axial flow near the blade tip for grooved case and hence increase in stall margin.

9. The mass flow through the tip gap is lowest for the swept rotor with grooved shroud. This has resulted in a lower mixing loss in case of swept rotor.

\section{Acknowledgements}

Authors hereby take this opportunity to thank Honeywell Technology Solutions, Bangalore, India, for allowing this research work to be published.

$\begin{array}{ll}\text { Greek Symbols } \\ A & \text { area }\left[\mathrm{m}^{2}\right] \\ C h & \text { chord length } \\ M c & \text { corrected mass flow rate }[\mathrm{kg} / \mathrm{s}] \\ M, m & \text { physical mass flow rate }[\mathrm{kg} / \mathrm{s}]\end{array}$

$P R \quad$ ratio of total pressure at outlet to that at inlet

$P T_{\text {in }} \quad$ inlet total pressure $[\mathrm{kPa}]$

$P T_{\text {out }} \quad$ outlet total pressure $[\mathrm{kPa}]$

$T R \quad$ ratio of total temperature at outlet to that at inlet

$T T_{\text {in }} \quad$ inlet total temperature $[\mathrm{K}]$

$V_{\mathrm{m}} \quad$ meridional velocity $[\mathrm{m} / \mathrm{s}]$

$\gamma \quad$ ratio of specific heats

$\phi \quad$ any variable

$\bar{\phi} \quad$ average value of variable

stall properties corresponding to stall point

OP properties corresponding to operating point

$\lambda \quad$ sweep magnitude

$\rho \quad$ density $\left[\mathrm{kg} / \mathrm{m}^{3}\right]$

\section{References}

[1] Breugalmans F 1987 Investigation of dihedral effects in compressor cascades. AGARD-CP-421, Massachusetts

[2] Sasaki T and Breugalmans F 1997 Comparison of sweep and dihedral effects on compressor cascade performance. $J$. Turbomach. 120: 454-463

[3] Inoue M, Kuroumaru M, Furukawa, M, Kinoue Y, Tanino T, Maeda S and Okuno K 1997 Controlled-Endwall-Flow blading for multistage axial compressor. In: Proceedings of the International Gas Turbine and Aeroengine Congress and Exhibition, vol. 1, p. V001T03A044, https://doi.org/10.1115/ 97-GT-248

[4] Godwin W R 1957 Effect of sweep on performance of compressor blade sections, as indicated by swept-blade rotor, unswept-blade rotor and cascade tests. Report NACA$\mathrm{TN}-4062$

[5] Wadia A R, Szucs P N and Crall D W 1998 Inner workings of aerodynamic sweep. J. Turbomach. 120: 671-682

[6] Choi K J, Kim J H and Kim K Y 2010 Design optimization of circumferential casing grooves for a transonic axial compressor to enhance stall margin. In: Proceedings of ASME GT 2010, vol. 7, pp. 687-695, https://doi.org/10.1115/ GT2010-22396

[7] Ramakrishna P V and Govardhan M 2009 Study of sweep and induced dihedral effects in sub-sonic axial flow compressor passages - part 1: design considerations-changes in incidence, deflection and streamline curvature. Int. J. Rotating Mach. 2009, https://doi.org/10.1155/2009/787145

[8] Biollo R and Benini E 2008 Aerodynamic behaviour of a novel three-dimensional shaped transonic compressor rotor blade. In: Proceedings of ASME GT 2008, vol. 6, pp. 695-706, https://doi.org/10.1115/GT2008-51397

[9] Bailey E E 1972 Effect of grooved casing treatment on the flow range capability of a single-stage axial-flow compressor. Report NASA $\_T M \backslash X \backslash$ 2459

[10] Urasek D C, Lewis Jr. G W and Moore R D 1976 Effect of casing treatment on performance of an inlet stage for a transonic multistage compressor. Report NASA _TM $\_\mathrm{X} \_3347$

[11] Lin F, Ning F and Liu H 2008 Aerodynamics of compressor casing treatment-part I: experiment and time-accurate numerical simulation. In: Proceedings of ASME GT 2008, vol. 6, pp. 731-744, https://doi.org/10.1115/GT2008-51541 
[12] Khan J A, Parvez K, Ahmad S and Mushtaq A 2011 Effect of circumferential grooves and tip recess on stall characteristics of transonic axial compressor rotor. In: Proceedings of the 49th AIAA Aerospace Sciences Meeting including the New Horizons Forum and Aerospace Exposition, https://doi.org/ 10.2514/6.2011-743

[13] Reid L and Moore R D 1978 Design and overall performance of four highly loaded, high-speed inlet stages for an advanced high-pressure-ratio core compressor. Report NASA TP 1337

[14] Reid L and Moore R D 1980 Experimental study of low aspect ratio compressor blading. J. Eng. Power 104: 875-882, https://doi.org/10.1115/1.3230353

[15] Hah C and Reid L 1992 A viscous flow study of shockboundary layer interaction, radial transport and wake development in a transonic compressor. J. Turbomach. 114: 538-547, https://doi.org/10.1115/1.2929177

[16] Suder K L and Celestina M L 1996 Experimental and computational investigation of the tip clearance flow in a transonic axial compressor rotor. J. Turbomach. 118: 218-229, https://doi.org/10.1115/1.2836629

[17] Hah C and Loellbach J 1999 Development of hub corner stall and its influence on the performance of axial compressor blade rows. J. Turbomach. 121: 67-77, https://doi.org/10. $1115 / 1.2841235$
[18] Chima R V 1998 Calculation of tip clearance effects in a transonic compressor rotor. J. Turbomach. 121: 131-140, https://doi.org/10.1115/1.2841374

[19] Gerolymos G A and Vallet I 1999 Tip-clearance and secondary flows in a transonic compressor rotor. J. Turbomach. 121: 751-762, https://doi.org/10.1115/1.2836729

[20] Yamada K, Furukawa M, Nakano T, Inoue M and Funazaki K 2004 Unsteady three-dimensional flow phenomena due to breakdown of tip leakage vortex in a transonic axial compressor rotor. In: Proceedings of ASME GT 2004, pp. 515-526, https://doi.org/10.1115/GT2004-53745

[21] Goswami S N and Govardhan M 2016 Effect of sweep on performance of an axial compressor with casing grooves. In: Proceedings of ASME GT 2016, p. V02AT37A004, 10 pp., https://doi.org/10.1115/GT2016-56045

[22] Ansys CFX®15.0 User Manuals

[23] Huang X, Chen H and Fu S 2008 CFD investigation on the circumferential grooves casing treatment of transonic compressor. In: Proceedings of ASME GT 2008, pp. 581-589, https://doi.org/10.1115/GT2008-51107

[24] Huang X, Chen H, Ke S and Fu S 2010 An analysis of the circumferential grooves casing treatment for transonic compressor flow. Sci. China Phys. Mech. Astron. 53(2): 353-359, https://doi.org/10.1007/s11433-010-0123-0 